\title{
Cost-effectiveness Analysis for Genotyping before Allopurinol Treatment to Prevent Severe Cutaneous Adverse Drug Reactions
}

\author{
Ching-Hua Ke, Wen-Hung Chung, Yen-Hsia Wen, Yaw-Bin Huang, Hung-Yi Chuang, \\ You-Lin Tain, Yu-Ching Lily Wang, Cheng-Chih Wu, and Chien-Ning Hsu
}

ABSTRACT. Objective. Patients with an HLA-B*58:01 allele have an increased risk of developing severe cutaneous adverse drug reactions (SCAR) when treated with allopurinol. Although one-off pharmacogenetic testing may prevent life-threatening adverse drug reactions, testing prior to allopurinol initiation incurs additional costs. The study objective was to evaluate the cost-effectiveness of HLA-B*58:01 screening compared with using other available urate-lowering agents (ULA).

Methods. A decision-analytical model was used to compare direct medical costs and effectiveness [including lifetime saved, quality-adjusted life-yrs (QALY) gained] in treating new patients with the following options: (1) genetic screening followed by allopurinol prescribing for noncarriers of HLA-B*58:01, (2) prescribing benzbromarone without screening, (3) prescribing febuxostat without screening, and (4) prescribing allopurinol without screening. A 1-year time frame and third-party payer perspective were modeled for both the entire cohort (base-case) and for the subgroup of patients with chronic kidney disease (CKD).

Results. The incremental cost-effectiveness ratio of genetic screening prior to ULA therapy was estimated as New Taiwan (NT) \$234,610 (US\$7508) per QALY gained in the base-case cohort. For patients with CKD, it was estimated as NT\$230,925 (US\$7390) per QALY. The study results were sensitive to the probability of benzbromarone/febuxostat-related hypersensitivity, and a negative predicted value of genotyping.

Conclusion. HLA-B*58:01 screening gave good value for money in preventing allopurinol-induced SCAR in patients indicated for ULA therapy. In addition to the costs of genotyping, it is important to monitor ULA safety closely in adopting HLA-B*58:01 screening in practice. (First Release April 1 2017; J Rheumatol 2017;44:835-43; doi:10.3899/jrheum.151476)

Key Indexing Terms:

ALLOPURINOL

HLA-B*58:01

SCAR

COST-EFFECTIVENESS ANALYSIS

CHRONIC KIDNEY DISEASE

Drug hypersensitivity carries an immense burden of morbidity and mortality ${ }^{1,2,3}$. According to the Taiwan National Drug Relief and Voluntary Adverse Drug Reporting System, allopurinol is the drug with the most potential to induce severe cutaneous adverse reactions (SCAR), including Stevens-Johnson syndrome (SJS), toxic epidermal necrolysis (TEN), and drug reaction with eosinophilia and systemic symptoms ${ }^{4,5}$. A strong association between possession of the HLA-B*58:01 allele and risk of allopurinol-induced SCAR has been observed among Han Chinese (i.e., ethnic Chinese from Taiwan and Hong Kong), Thais, Indians, and Koreans ${ }^{6,7}$. The prevalence of the HLA-B*58:01 allele ranges from $1.9 \%$ to $27.5 \%$ in the general Asian population, but is very rare in whites ${ }^{8}$. Chronic kidney disease (CKD) is
From the School of Pharmacy, and Department of Public Health and Center of Excellence for Environmental Medicine, Kaohsiung Medical University; Department of Pharmacy, Kaohsiung Medical University, Chung-Ho Memorial Hospital; Department of Pediatrics, and Department of Pharmacy, Kaohsiung Chang Gung Memorial Hospital, Kaohsiung; Department of Dermatology, Drug Hypersensitivity Clinical and Research Center, Chang Gung Memorial Hospital, College of Medicine, Chang Gung University, Taoyuan, Taiwan.

Funded by Kaohsiung Chang Gung Memorial Hospital (BMRPD36).

C.H. Ke, MSc, School of Pharmacy, Kaohsiung Medical University; W.H. Chung, MD, PhD, Department of Dermatology, Drug Hypersensitivity Clinical and Research Center, Chang Gung Memorial Hospital, College of Medicine, Chang Gung University; Y.H. Wen, PhD, School of Pharmacy, Kaohsiung Medical University; Y.B. Huang, PhD, School of Pharmacy, Kaohsiung Medical University, and Department of
Pharmacy, Chung-Ho Memorial Hospital; H.Y. Chuang, MD, PhD, Department of Public Health and Center of Excellence for Environmental Medicine, Kaohsiung Medical University; Y.L. Tain, MD, PhD, Department of Pediatrics, Kaohsiung Chang Gung Memorial Hospital, College of Medicine, Chang Gung University; Y.C. Wang, MSc, Department of Pharmacy, Kaohsiung Chang Gung Memorial Hospital; C.C.Wu, MSc, Department of Pharmacy, Kaohsiung Chang Gung Memorial Hospital; C.N. Hsu, PhD, School of Pharmacy, Kaohsiung Medical University, and Department of Pharmacy, Kaohsiung Chang Gung Memorial Hospital.

Address correspondence to C.N. Hsu, Department of Pharmacy, Kaohsiung Chang Gung Memorial Hospital, 123, Dabi Road, Kaohsiung, Taiwan 833.E-mail: chien_ning_hsu@hotmail.com

Full Release Article. For details see Reprints and Permissions at jrheum.org. Accepted for publication February 3, 2017.

Personal non-commercial use only. The Journal of Rheumatology Copyright @ $\odot 2017$. All rights reserved 
also suggested to be a nongenetic factor associated with allopurinol hypersensitivity symptoms ${ }^{9,10}$. A plausible mechanism for this is that the oxypurinol metabolite accumulates in patients with impaired renal function ${ }^{11}$ so that the risk of allopurinol hypersensitivity is increased by $4-5 x$ in patients with CKD treated with allopurinol (OR 4.7, 95\% CI 2.3-9.3 $)^{11}$.

The 2012 American College of Rheumatology (ACR) gout guidelines recommends xanthine oxidase inhibitor (XOI) therapy with either allopurinol or febuxostat, and probenecid as the first-line alternative where XOI is contraindicated $^{12}$. Other urate-lowering agents (ULA) such as benzbromarone are also in common use in Taiwan ${ }^{13}$. Benzbromarone is used particularly in patients with renal function disorders. Its recommended dose is usually 50-200 $\mathrm{mg}$ daily, which is generally well tolerated, although regular liver function monitoring is essential ${ }^{14}$. Specific genotyping for HLA-B*58:01 is currently available in Taiwan. Although the Taiwan Food and Drug Administration has suggested genotyping patients before prescribing allopurinol since $2009^{15}$, this genotyping is not reimbursed by the National Health Insurance (NHI) program. Although the cost of genotyping is high, the high cost of alternative ULA and the chance that many patients will not develop SCAR mean that the value of routine genotyping prior to prescribing allopurinol is unclear.

We therefore evaluated the cost-effectiveness of HLA-B*58:01 screening compared with using available alternative ULA (benzbromarone, febuxostat, and allopurinol) without prior genotyping. We also examined the incidence of allopurinol-related SCAR and the associated costs of health services use.

\section{MATERIALS AND METHODS}

Identification of patients with allopurinol-related SCAR. A retrospective, population-based cohort study was conducted to identify incidence, mortality, and total medical costs attributed to allopurinol-related SCAR, using the 2 million random beneficiaries from longitudinal database in the Collaboration Center of Health Information Application (CCHIA), which were available from 2000 to 2011. The CCHIA database holds complete information about almost all claims from the Taiwan NHI program, and its details have been well described elsewhere ${ }^{16}$. The study proposal was reviewed and approved by the institutional review board and ethics committee of Chang Gung Medical Foundation in Taiwan (103-4218B).

Analysis of incidence of SCAR. The initial adverse drug reaction-related SCAR visits were defined by the following criteria: (1) International Classification of Diseases, 9th ed codes (Supplementary Table 1, available with the online version of this article) for SCAR from outpatient or emergency visit or hospitalization discharge ${ }^{17,18}$, (2) allopurinol discontinuation following the diagnosis of SCAR, and (3) SCAR events occurring within 100 days of discontinuation ${ }^{18}$. Because HLA-B*58:01 is not only a risk factor for severe cutaneous reactions, but also for mild cutaneous adverse reactions, such as maculopapular exanthema in the Han Chinese population ${ }^{19}$, broad criteria of any allopurinol-related skin reactions were used to minimize underestimation of incidence. The total number of incident cases over the study period was consistent with the documented reports in the national reporting center of adverse drug reactions in Taiwan between 1999 and $2010^{4}$.
The incidence of adverse drug reactions was calculated for each calendar year from 2001 to 2011. Incidence was expressed as the number of SCAR divided by the total number of new allopurinol users in each calendar year. Within the cohort of allopurinol-related SCAR, the incidence of death and discharge were calculated using the number of adverse drug reactions in the corresponding calendar year as the denominator. The subgroup analysis was conducted for those new allopurinol users with CKD, assessing the incidence of SCAR in the same way.

All costs for outpatient care, emergency visits, and inpatient medical care are paid by a single third-party payer NHI program in Taiwan. The economic perspective for our study, therefore, included all related healthcare costs. The burden of adverse drug reaction-related visits to the healthcare system was estimated among patients receiving healthcare services for allopurinol-associated adverse reactions. The total cost included all corresponding outpatient and emergency visits and any hospital admissions. The total medical costs were updated to the last observed period of data and expressed in 2015 Taiwan dollars. The database was censored at the study end date, so costs could not be estimated for patients with SCAR after September 30, 2011. Mean annual costs of allopurinol-related SCAR, direct medical cost per SCAR event, and overall medical costs between 2001 and 2011 were assessed.

Cost-effectiveness analysis (CEA). A decision tree model was developed to estimate the cost-effectiveness of HLA-B*58:01 genotyping for adult patients with newly diagnosed hyperuricemia or gout in Taiwan for whom allopurinol, benzbromarone, or febuxostat were considered suitable as first-line monotherapy (Figure 1).

Model variables. Prior cost-effectiveness analyses of HLA-B*58:01 genetic test did not include comparisons with febuxostat and benzbromarone as alternative treatment strategy ${ }^{20,21}$. In accordance with updated treatment guidelines for hyperuricemia and the pattern of ULA use in the real-world setting, the treatment strategies evaluated in our present study included (1) genetic screening prior to allopurinol prescribing, (2) prescribing benzbromarone without screening, (3) prescribing febuxostat without screening, and (4) prescribing allopurinol without screening. Table 1 presents variables used in the model ${ }^{8,9,11,22,23,24,25,26,27}$.

Because the 2012 ACR gout guidelines recommended that allopurinol and febuxostat be considered as equivalent first-line options, the efficacy variation of ULA because of individual patients' serum uric acid level and escalating doses were not considered in our study ${ }^{12}$. Overall, febuxostat was generally well tolerated and equally safe with allopurinol in large clinical trials, also in patients with renal dysfunction ${ }^{28,29}$.

Benzbromarone is considered at least equally effective as allopurinol at achieving target serum uric acid ${ }^{14}$, particularly for patients in whom allopurinol has insufficient response or toxicity, which is consistent with the recommendations of European League Against Rheumatism in $2016^{30}$ Benzbromarone was withdrawn from the French market in 2003 by the manufacturer following the spontaneous reports of serious liver toxicities; it is still available in some European countries and several Asian countries, including Taiwan. The incidence of benzbromarone-induced hepatotoxicity has been estimated to be 1:17,000 persons based on 4 published case reports and 11 cases of postmarket safety reports between 1994 and $2002^{31}$. The benefit-risk assessment of benzbromarone in the treatment of gout does not support the decision for the market withdrawal ${ }^{31}$. The current labeling of benzbromarone in Taiwan lists liver cirrhosis as contraindication and its risk of hepatotoxicity is lower than the general incidence of antituberculosis $(5.9 / 1000 \text { person-mos) })^{32}$ and antifungal (1.58-8.39 per 10,000) $)^{33}$ drug-induced hepatoxicity. Benzbromarone was modeled in our study, and even though use of benzbromarone has changed over time ${ }^{13}$, it remains a common choice of ULA in a clinical setting.

Patients who have a negative HLA-B*58:01 testing result could receive and remain with allopurinol therapy. Patients with a benzbromarone strategy could switch to febuxostat (13.75\%) if they experienced intolerance or failed to respond, or could remain with benzbromarone within 1 year of initial therapy based on our own investigation in the study setting. Further, patients who began with febuxostat could remain in the therapy for over 1 year. The

Personal non-commercial use only. The Journal of Rheumatology Copyright @ $\odot 2017$. All rights reserved 


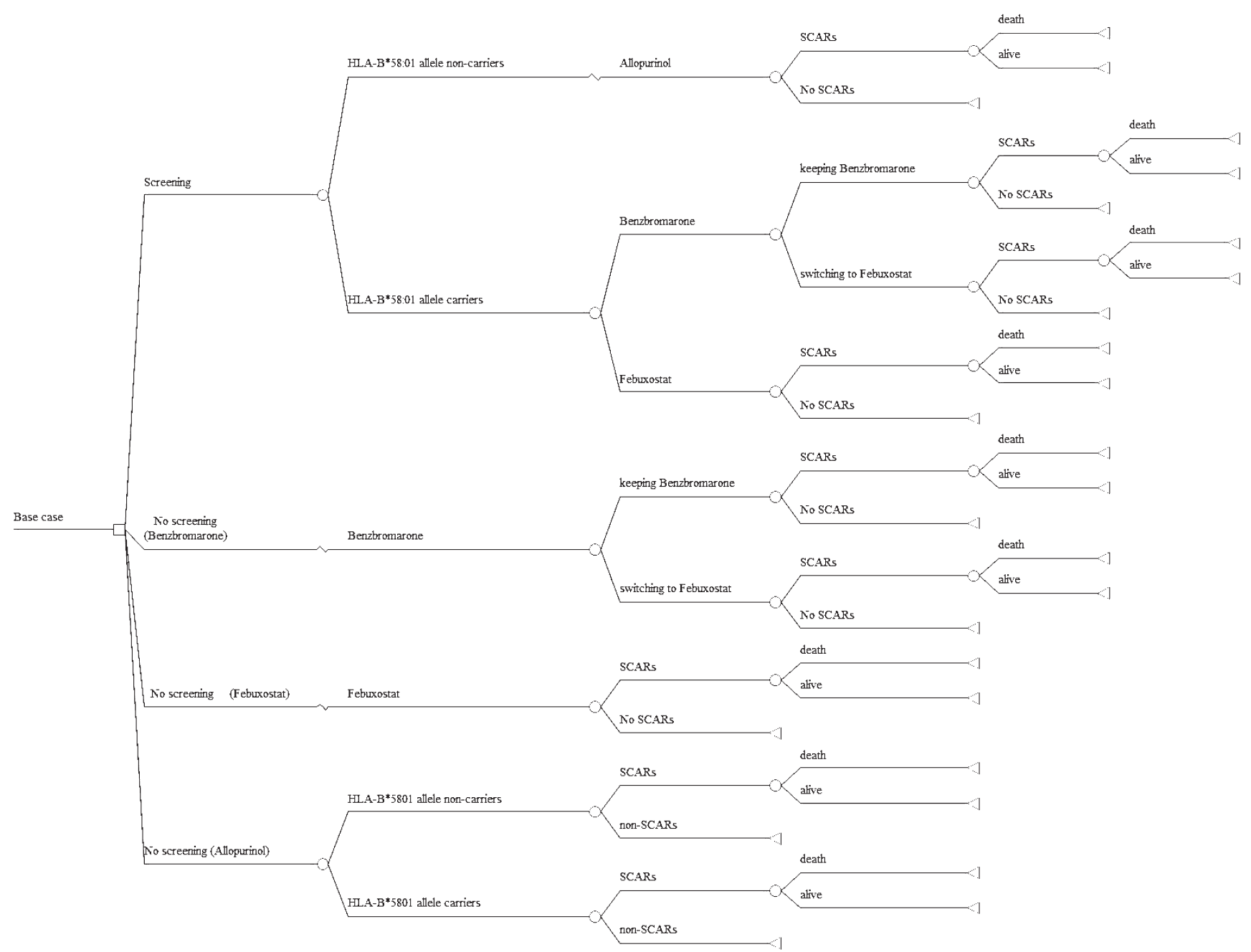

Figure 1. Decision tree model for treatment strategy in allopurinol new users. There were 4 treatment strategies in the model: (1) genetic screening prior to allopurinol prescribing, (2) prescribing benzbromarone without screening, (3) prescribing febuxostat without screening, and (4) prescribing allopurinol without screening. Three possible outcomes were modeled: (1) being cured of allopurinol-induced SCAR, (2) death attributed to allopurinol-induced SCAR, and (3) not developing allopurinol-induced SCAR. SCAR: severe cutaneous adverse drug reactions.



Figure 2. One-way sensitivity analyses in the base-case cohort. One-way sensitivity analysis results showed the influence of individual variables on the ICER of the genetic screening strategy compared with no screening with benzbromarone. The minus sign at the left bar indicates that ICER decreases as the variables increase. ICER: incremental cost-effectiveness ratio; SCAR: severe cutaneous adverse drug reactions; ULA: urate-lowering agents. 
Table 1. The variables in the cost-effectiveness analysis model.

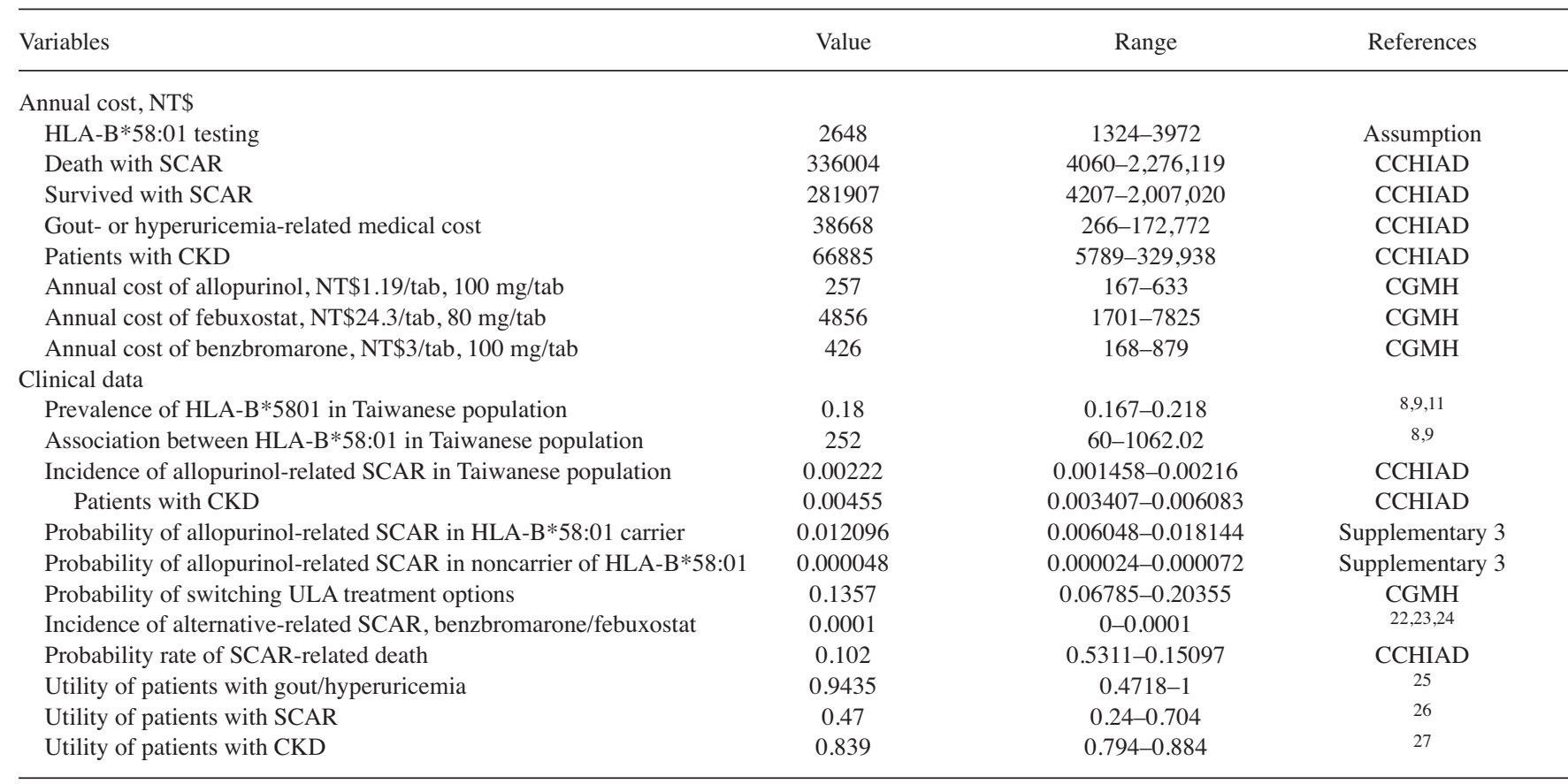

NT\$: New Taiwan Dollar (US\$1 = NT\$31.25 in 2015); SCAR: severe cutaneous adverse reactions; CKD: chronic kidney disease; ULA: urate-lowering agent; CCHIAD: Collaboration Center of Health Information Application database; CGMH: Chang Gung Memorial Hospital.

clinical course of action for patients receiving ULA therapy was modeled in our study to reflect actual decision making in a clinical practice setting. A combination ULA therapy strategy (e.g., allopurinol or febuxostat combined with benzbromarone) was not considered during the initial phase of treatment.

Probenecid and sulfinpyrazone are uricosuric agents that were recommended as second-line use in those resistant to or intolerant of allopurinol $^{30,34,35}$, but both drugs were not commonly used $(<50 / 100,000$ persons) and not considered in the model ${ }^{13}$.

Incidence of SCAR. The probability of allopurinol-induced SCAR in patients with/without HLA-B $* 58: 01$ was calculated using conditional probabilities, in line with a previous study (Supplementary Data 1, available with the online version of this article $)^{21,22}$. Although benzbromarone- and febuxostat-related severe skin adverse reactions have been reported, their actual incidence was not clear ${ }^{23,24}$. We therefore assumed the probability of an adverse drug reaction was 0.0001 ( 1 per 10,000 persons) or "very rare"36.

Outcomes. Three possible outcomes were defined and modeled. These were (1) being cured of allopurinol-induced SCAR, (2) death attributed to allopurinol-induced SCAR, and (3) not developing allopurinol-induced SCAR. The utility for patients with gout has been measured as 0.9435 for chronic patients in the Japanese population ${ }^{25}$. The utility for patients who developed SCAR was measured as 0.47 in the Thai population ${ }^{26}$. For patients with CKD, utility was recommended as $0.839(0.794-0.884)$ in the Japanese population $^{27}$.

The results were presented as the total expected costs, lifetime saved, and quality-adjusted life-years (QALY) gained. The incremental cost-effectiveness ratio was expressed as incremental cost per life-years or QALY gained.

Time frame. Because allopurinol-induced SCAR mostly occur within 1 year of the initiation of treatment, and $90 \%$ of cases occur within 180 days ${ }^{18}$, a 1 -year study time frame was set. Costs of ULA therapy were followed for 1 year. The 1-year prescription cost of ULA use was measured based on the medication cost as unit cost of drug multiplied by annual quantity ${ }^{37}$.
The model further assumed that the cost of the HLA-B*58:01 screening test was NT $\$ 2648$ (US\$87.7, US\$1 = NT $\$ 31.25$ in 2015), the price given in the Taiwan National Health Insurance program.

Sensitivity analysis. Our model included data from various sources, so it was important to validate the data. We performed univariate deterministic analyses with a Tornado diagram for the variables in the model: the incidence of SCAR related to ULA alternatives, the association between the HLA-B*58:01 allele and allopurinol-related SCAR, positive predictive value and negative predictive value (NPV) of the HLA-B*58:01 genetic test (Supplementary Data 1, available with the online version of this article), and the prevalence of the HLA-B* 58:01 allele in the Taiwanese population. Also analyzed were the probability of death from allopurinol-related SCAR, the utility for patients with gout and with SCAR, the cost of HLA-B 58:01 screening, the annual cost of ULA (allopurinol, febuxostat, or benzbromarone) and hyperuricemia/gout management, the probability and duration of ULA switching, and the total medical costs of treating patients with and dying from SCAR. The range of each variable was retrieved from the $95 \%$ $\mathrm{CI}$, if available. If not available, the range was defined as $0.5-1.5 \times$ the base value.

A probabilistic sensitivity analysis (PSA) was performed to obtain cost-effectiveness acceptability curves at different maximum willingness to pay (WTP) thresholds. The PSA examined the effect of uncertainty in all variables simultaneously using a second-order Monte Carlo simulation across 10,000 iterations, with the value of each variable being determined by a random draw from an assigned distribution.

The same analysis was conducted for the entire sample of patients and for the subgroup of patients with CKD. All assumptions were presented to and validated by clinical and health economic experts. A decision tree model was developed in TreeAge Pro 2014 software (TreeAge Inc.) to estimate the cost-effectiveness of HLA-B*5801 genotyping prior to ULA therapy.

Statistical analysis. Variables were expressed as means and SD or median and interquartile range (IQR) for continuous variables, and numbers and percentages for categorical variables. Statistical tests of significance for differences used the Pearson chi-square test for dichotomous variables and 
the Student $t$ test for continuous variables. The level of significance was set at $p<0.05$. All data processing and analyses were conducted using SAS 9.3 (SAS Inc.)

\section{RESULTS}

Health outcomes of allopurinol-related SCAR. Supplementary Table 2 (available with the online version of this article) shows the incidence of allopurinol-related SCAR and lifetime saved. A total of 147 allopurinol-related SCAR were observed among 66,276 new allopurinol users between January 2001 and December 2011. The mean time of followup was 28.08 months, and the overall incidence of allopurinol-related SCAR was $0.22 \%$ (95\% CI $0.19-0.26 \%$ ) or 2.2 SCAR cases per 1000 allopurinol new users. Of allopurinol-related SCAR $(n=147), 15$ patients $(10.2 \%)$ died during hospitalization for SCAR (95\% CI 5.3-15.1\%). The average length of time from starting the treatment to SCAR-related death was 297.98 days (median 304 days, IQR 296-310 days). In patients with CKD, the incidence of allopurinol-related SCAR was $0.46 \%$ (95\% CI $0.34-0.61 \%$ ) in the study population. Characteristics of patients are shown in Supplementary Table 3 (available with the online version of this article).

Costs. Supplementary Table 4 (available with the online version of this article) shows the total medical costs for patients with allopurinol-related SCAR. The average medical costs for each allopurinol-related SCAR hospitalization was NT\$113,285 (median NT\$40,760, IQR NT\$17,309-113,562). For the 132 patients who survived SCAR, the mean medical costs were NT\$92,091 (median NT\$31,119, IQR NT \$47,393-311,939). The medical costs increased in the 15 deceased patients, with mean cost being NT\$264,140 (median NT\$140,151, IQR NT\$67,567-224,192).
Results of cost-effectiveness analysis. Table 2A shows the results of the base-case analysis. Two strategies dominated: febuxostat without genotyping (i.e., higher price), and allopurinol without genotyping (i.e., less effective). Compared with benzbromarone without screening, genetic screening with allopurinol had an additional cost of NT\$2620, but added 0.00012 life-years and 0.01117 QALY. The estimated incremental cost-effectiveness ratio (ICER) of genetic testing was NT $\$ 21,651,688$ per life-year saved and NT $\$ 234,610$ per QALY gained.

The results for patients with CKD are shown in Table 2B. Compared with benzbromarone without screening, genetic testing added NT\$2195, 0.00014 life-years, and 0.01089 QALY. The estimated ICER of genetic testing was NT $\$ 17,378,777$ per life-year and NT\$230,925 per QALY.

Figure 2 shows the results of the 1-way sensitivity analyses on the structural assumptions. These sensitivity analyses indicated that the ICER was sensitive to several assumptions: the incidence of SCAR following ULA alternatives, the utility of patients with gout/hyperuricemia, the NPV of HLA-B*58:01 screening, and the cost of genetic testing. When the incidence of SCAR from ULA alternatives in the Taiwanese population was varied from 0 to 0.0001 , the ICER was -NT $\$ 1,684,609$ to NT\$11,945,417 per QALY gained.

Figure 3 shows probability sensitivity analyses. Genetic testing increased both costs and QALY in the majority of iterations (Figure 3A). Figure 3B shows the acceptability curves for HLA-B*58:01 screening prior to treatment. In the probability sensitivity analysis for the base-case cohort, genetic screening revealed that $70 \%$ of the iterations were cost-effective at a WTP of NT $\$ 800,000$ (US $\$ 25,600$ in 2015) per additional QALY gained in Taiwan. For patients with

Table 2A. Cost-effectiveness analysis in the base-case cohort.

\begin{tabular}{lccccccccc}
\hline Strategy & $\begin{array}{c}\text { Cost, } \\
\text { NT\$ }\end{array}$ & $\begin{array}{c}\text { Incremental } \\
\text { Cost, NT\$ }\end{array}$ & Life-yrs & $\begin{array}{c}\text { Incremental } \\
\text { Life-yrs }\end{array}$ & $\begin{array}{c}\text { ICER, } \\
\text { NT\$/life-yrs }\end{array}$ & $\begin{array}{c}\text { QALY } \\
\text { Incremental } \\
\text { QALY }\end{array}$ & $\begin{array}{c}\text { ICER, } \\
\text { NT \$QALY }\end{array}$ \\
\hline No screening, benzbromarone & 39,419 & - & 0.99841981 & - & - & 0.943448 & - \\
No screening, allopurinol & 39,476 & 57 & 0.997782 & -0.000638 & Dominated & 0.940743 & -0.002705 & Dominated \\
Screening & 42,040 & 2620 & 0.99854081 & 0.000121 & $21,651,668.62$ & 0.9454617 & 0.011169 & $234,610.94$ \\
No screening, febuxostat & 43,548 & 1509 & 0.99841981 & 0.000121 & Dominated & 0.943448 & -0.011169 & Dominated \\
\hline
\end{tabular}

NT\$: New Taiwan Dollar (US\$1 = NT\$31.25 in 2015); ICER: incremental cost-effectiveness ratio; QALY: quality-adjusted life-years.

Table 2B. Cost-effectiveness analysis in the subgroup of patients with chronic kidney disease.

\begin{tabular}{lccccccrr}
\hline Strategy & $\begin{array}{c}\text { Cost, } \\
\text { NT\$ }\end{array}$ & $\begin{array}{c}\text { Incremental } \\
\text { Cost, NT\$ }\end{array}$ & Life-yrs & $\begin{array}{c}\text { Incremental } \\
\text { Life-yrs }\end{array}$ & $\begin{array}{c}\text { ICER, } \\
\text { NT\$/life-yrs }\end{array}$ & $\begin{array}{c}\text { QALY } \\
\text { Incremental } \\
\text { QALY }\end{array}$ & $\begin{array}{c}\text { ICER, } \\
\text { NT\$/QALY }\end{array}$ \\
\hline No screening, benzbromarone & 67,706 & - & 0.99841981 & - & - & 0.791553 & - \\
No screening, allopurinol & 68,144 & 439 & 0.99545 & -0.0029698 & Dominated & 0.789606 & -0.001947 & Dominated \\
Screening & 69,901 & 2195 & 0.99856458 & 0.00014477 & $17,378,777.28$ & 0.802447 & 0.010894 & $230,925.51$ \\
No screening, febuxostat & 71,763 & 1862 & 0.99841981 & -0.00014477 & Dominated & 0.791553 & -0.010894 & Dominated \\
\hline
\end{tabular}

NT\$: New Taiwan Dollar (US\$1 = NT\$31.25 in 2015); ICER: incremental cost-effectiveness ratio; QALY: quality-adjusted life-years.

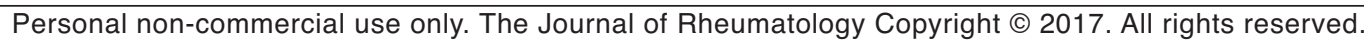




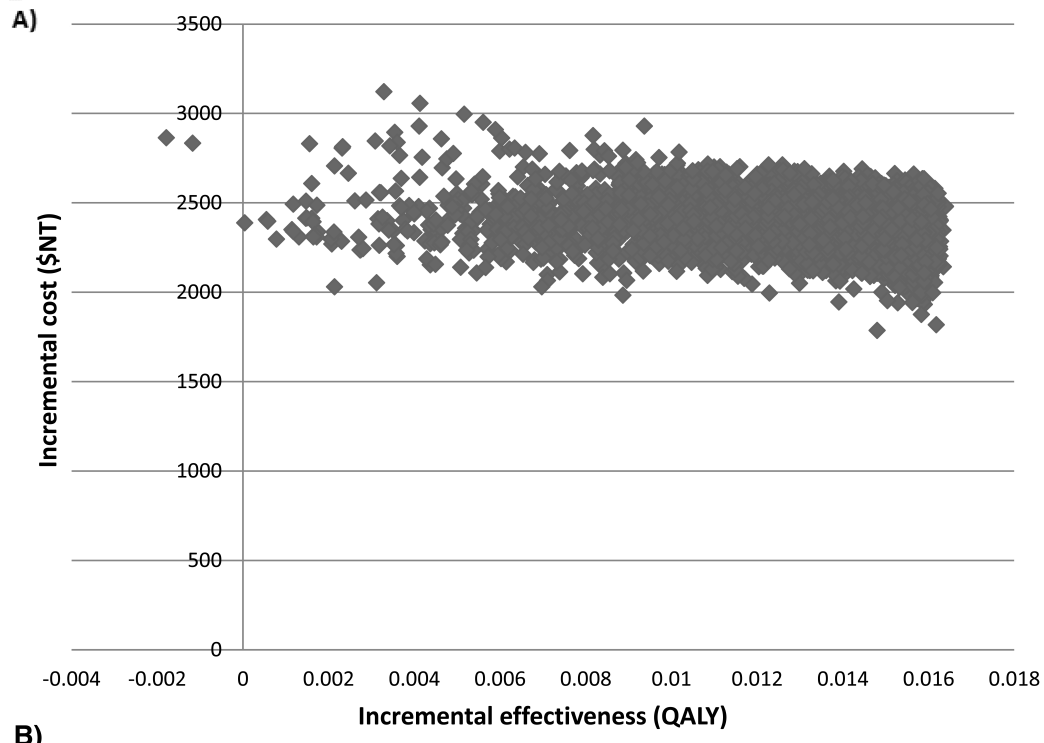

B)

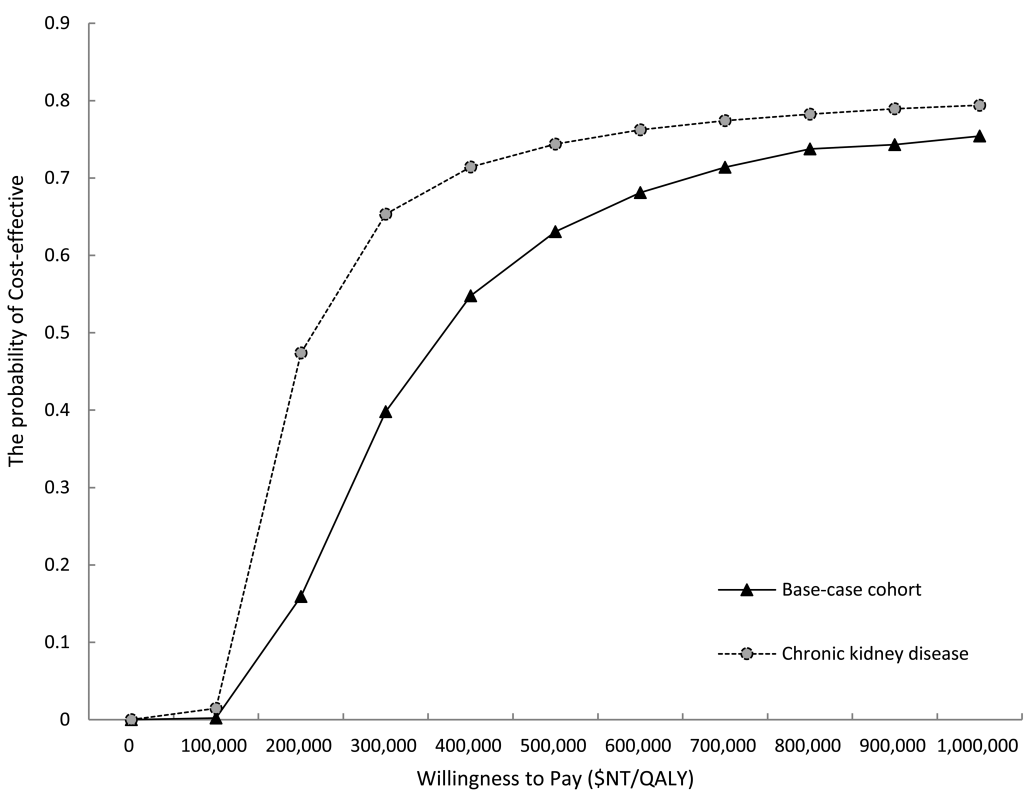

Figure 3. (A) Cost-effectiveness scatter plot. Each point represents incremental cost (2015 values) and QALY between no screening with febuxostat and no screening with benzbromarone therapy from Monte Carlo simulation with multiple model variables. (B) Cost-effectiveness acceptability curve of genetic screening strategy in base-case cohort and patients with CKD. Cost-effectiveness acceptability curves from the probabilistic sensitivity analysis represent the 10,000 iterations. The probability of genotyping being cost-effective is $70 \%$ at a willingness-to-pay over NT $\$ 800,000$ (US $\$ 25,600$ ) per additional QALY gained in the base-case cohort, and NT $\$ 400,000$ (US $\$ 12,800$ ) per QALY in patients with CKD. CKD: chronic kidney disease; QALY: quality-adjusted life-years; $\$ N T=$ New Taiwan Dollar $($ US $\$ 1=$ NT $\$ 31.25$ in 2015).

CKD, the probability of cost effectiveness was $70 \%$ at a WTP of NT $\$ 400,000$ (US $\$ 12,800$ in 2015) per QALY. The probability of HLA-B*58:01 screening being cost-effective increased following an increase in WTP.

The number needed to screen was calculated to assess the effect of HLA-B*58:01 screening at population level. Of allopurinol users, 461 and 225 patients needed to be screened for HLA-B*58:01 to prevent 1 case of SCAR in the base-case cohort and among patients with CKD, respectively (Supplementary Table 5, available with the online version of this article). Thus, the cost of preventing 1 case of SCAR was calculated to be NT $\$ 1,220,728$ for 461 base-case patients, 
and NT $\$ 595,800$ for 225 patients with CKD (using a cost of NT\$2648 for HLA-B*58:01 screening).

\section{DISCUSSION}

Our study objective was to inform health professionals and policymakers about the cost-effectiveness of genetic screening for preventing allopurinol-related SCAR. Our analysis incorporated the burden of hypersensitivity derived from real-world data and clinical alternatives (benzbromarone and febuxostat) in the model. The base-case analysis, based on available data and most likely clinical assumptions, indicated that HLA-B*58:01 screening prevented SCAR and saved more lives. Although no WTP threshold is universally accepted in Taiwan, the ICER per QALY of genetic screening prior to ULA therapy remained lower than the World Health Organization WTP threshold of US\$22,635-67,905 (1-3× gross domestic product per capita, US $\$ 22,635$ in 2015$)^{38,39}$, and is considered more cost-effective than alternatives without prior screening in Taiwan.

Hyperuricemia is prevalent in patients with $\mathrm{CKD}^{40}$. It is recommended that allopurinol therapy be started with a low dose and close monitoring in these patients, but the reduction in risk of allopurinol-related SCAR was not significant ${ }^{41,42}$. According to the incidence estimated by our study, the ICER of HLA-B*5801 screening was lower than for the base-case cohort compared with the same alterative treatment strategies. These study findings suggested that HLA-B*58:01 allele screening before ULA initiation is necessary to prevent potential life-threatening SCAR in high-risk subgroups.

The ICER in our study was sensitive to the safety profile of benzbromarone and febuxostat. Febuxostat has a chemical structure unrelated to allopurinol, and the probability of developing cross-related adverse drug reactions is theoretically low. The incidence of $2 \%$ rash was, however, documented among patients taking $80 \mathrm{mg} /$ day of febuxostat in a clinical trial, a level similar to that for allopurinol 300 $\mathrm{mg} / \mathrm{day}^{43}$. Since 2008 , several reports about tolerability and hypersensitivity to febuxostat have been published ${ }^{23,44,45}$. Although the immunological causality and true incidence of hypersensitivity reactions remain unclear, we suggest that scenarios linked to febuxostat hypersensitivity including renal insufficiency ${ }^{45}$ and severe skin reactions ${ }^{44}$ described in these reports are relevant to our study cohort. Our study's findings suggest the importance of gathering further evidence about patterns of febuxostat-related hypersensitivity reactions to strengthen the value of febuxostat initiation without genetic information in the target patient population. The price of febuxostat is another influential factor in its initiation.

Although there is a strong genetic association between allopurinol-related SCAR and the presence of HLA-B*58:01 allele, test results must be reliable to make genetic information meaningful for clinical decision making. Based on the prevalence of genetic markers in the population (18\%) and estimated incidence of allopurinol-related SCAR
(2.2/1000 persons), we calculated that 461 patients needed to be tested for HLA-B*58:01 to prevent 1 case of SCAR. Although the sensitivity and specificity of the HLA-B*58:01 test were reported to be $100 \%$, any possibility of an NPV for the test increased the ICER value per QALY gained.

The economic model was based on the best evidence available, but some limitations remained. First, from an economic perspective, it was necessary to consider a longer period of time to avoid giving excessive relevance to the direct treatment costs associated with one-off genotype screening under assessment. The main goal of our study was to compare the costs, health outcomes, and value of HLA-B*58:01 allele screening for allopurinol-related SCAR prevention. Our study did not consider the lifelong direct costs of care for SCAR-associated complications or benefits gained by avoidance of lifelong complications, such as blindness ${ }^{46,47}$, which may lead to underestimation of the value of one-off genotyping. For instance, the value of HLA-B*58:01 screening was concluded to be cost-effective with a lifelong time frame from a societal perspective in the Thai population ${ }^{21}$. On the other hand, inevitably additional uncertainties in both benefits and costs exist in relation to extrapolating evidence over time because of the implicit assumption that outcomes in the short term are good predictors of outcomes in longterm gout therapy. Another study evaluated the cost-effectiveness of HLA-B $* 5801$ over a lifetime horizon, but yielded contradictory conclusions on the longterm value in prevention of allopurinol-induced $\mathrm{SJS}_{\mathrm{TEN}}{ }^{20}$. It is possible that the assumptions of ULA outcomes and costs of gout management increased additional temporal uncertainties in the CEA models. Although the study design differed from ours in many ways, the short-term value of HLA-B*58:01 screening-guided therapy is consistent with that found in the Korean population ${ }^{48}$.

Second, the actual health utility among patients with hyperuricemia or gout in the initial stages of treatment is unknown. To show differences in value of genetic screening for prevention of rare but life-threatening adverse drug reaction, additional assumptions of utility for patients with SCAR and gout/hyperuricemia were made in our study. Sensitivity analyses revealed that if the utility for patients with ULA therapy remained $>0.53$ within the initial stage of treatment, genotyping can be considered cost-effective. Although we used an assumption of switching treatment in the model, in reality health-related quality of life may be considerably different from the base-case value. Further research on this topic is recommended to focus on the health utility for patients with hyperuricemia or gout to ensure the estimated value of genotyping is correct in practice.

Finally, because each country has a different healthcare system and ethnic background, our study results may only be applicable to specific populations, where HLA-B*58:01 is prevalent in the general population and the genetic screening resource is accessible. It is still not clear whether allopurinol

Personal non-commercial use only. The Journal of Rheumatology Copyright @ 2017 . All rights reserved. 
hypersensitivity is dose-dependent. Irrespective of genotyping, therefore, all patients should be assessed for risks attributed to allopurinol-related hypersensitivity reactions and closely monitored for signs and symptoms of hypersensitivity.

Our study demonstrated that genotyping can serve as a clinical decision-making support tool to prevent allopurinol-related SCAR, and indicated that HLA-B*58:01 screening is cost-effective compared with prescribing an alternative ULA (i.e., benzbromarone, febuxostat, and allopurinol) option without prior genotyping. Our study results highlight the need for genetic information to prevent severe hypersensitivity reactions and of developing effective interventions to identify and manage patients who are at risk of such life-threatening adverse reactions.

\section{ACKNOWLEDGMENT}

The authors thank Ying-Jen Hsu and SAS Working Group in the Department of Management of Information Systems, Chang Gung Memorial Foundation, for their technical assistance with electronic healthcare data retrieval. This study was based in part on data from the 2 million random beneficiaries from the longitudinal database (2000-2011) provided by the Ministry of Health and Welfare in Taiwan (Registered number H104006). We express our appreciation to the staff at the Collaboration Center of Health Information Application in Kaohsiung Medical University who assisted with population-based longitudinal database analysis.

\section{ONLINE SUPPLEMENT}

Supplementary material accompanies the online version of this article.

\section{REFERENCES}

1. Dechanont S, Maphanta S, Butthum B, Kongkaew C. Hospital admissions/visits associated with drug-drug interactions: a systematic review and meta-analysis. Pharmacoepidemiol Drug Saf 2014;23:489-97.

2. Suh DC, Woodall BS, Shin SK, Hermes-De Santis ER. Clinical and economic impact of adverse drug reactions in hospitalized patients. Ann Pharmacother 2000;34:1373-9.

3. Pirmohamed M, James S, Meakin S, Green C, Scott AK, Walley TJ, et al. Adverse drug reactions as cause of admission to hospital: prospective analysis of 18820 patients. BMJ 2004;329:15-9.

4. Taiwan Drug Relief Foundation. [Drug safety newsletter. National reporting center of adverse drug reaction in Taiwan, 2012]. [Website in Chinese]. [Internet. Accessed February 17, 2017.] Available from: www.tdrf.org.tw/ch/03_message/mes_03_list.asp

5. Taiwan Drug Relief Foundation. Drug safety newsletter. [Internet. Accessed February 10, 2017.] Available from: www.tdrf.org.tw/ch/03_message/mes_03_list.asp

6. Somkrua R, Eickman EE, Saokaew S, Lohitnavy M, Chaiyakunapruk N. Association of HLA-B*5801 allele and allopurinol-induced Stevens Johnson syndrome and toxic epidermal necrolysis: a systematic review and meta-analysis. BMC Med Genet 2011;12:118.

7. Zeng M, Zhang M, Liu F, Yan W, Kong Q, Sang H. Drug eruptions induced by allopurinol associated with HLA-B $* 5801$. Indian J Dermatol Venereol Leprol 2015;81:43-5.

8. Pavlos R, Mallal S, Phillips E. HLA and pharmacogenetics of drug hypersensitivity. Pharmacogenomics 2012;13:1285-306.

9. Zineh I, Mummaneni P, Lyndly J, Amur S, La Grenade LA, Chang $\mathrm{SH}$, et al. Allopurinol pharmacogenetics: assessment of potential clinical usefulness. Pharmacogenomics 2011;12:1741-9.

10. Jung JW, Song WJ, Kim YS, Joo KW, Lee KW, Kim SH, et al.
HLA-B58 can help the clinical decision on starting allopurinol in patients with chronic renal insufficiency. Nephrol Dial Transplant 2011;26:3567-72.

11. Chung WH, Chang WC, Stocker SL, Juo CG, Graham GG, Lee $\mathrm{MH}$, et al. Insights into the poor prognosis of allopurinol-induced severe cutaneous adverse reactions: the impact of renal insufficiency, high plasma levels of oxypurinol and granulysin. Ann Rheum Dis 2015;74:2157-64.

12. Khanna D, Fitzgerald JD, Khanna PP, Bae S, Singh MK, Neogi T, et al; American College of Rheumatology. 2012 American College of Rheumatology guidelines for management of gout. Part 1 : systematic nonpharmacologic and pharmacologic therapeutic approaches to hyperuricemia. Arthritis Care Res 2012;64:1431-46.

13. Cheng CL, Chao PH, Hsu JC, Weng MM, On AW, Yang YH. Utilization patterns of antihyperuricemic agents following safety announcement on allopurinol and benzbromarone by Taiwan Food and Drug Administration. Pharmacoepidemiol Drug Saf 2014;23:309-13.

14. Kydd AS, Seth R, Buchbinder R, Edwards CJ, Bombardier C. Uricosuric medications for chronic gout. Cochrane Database Syst Rev 2014:CD010457.

15. Taiwan Drug Relief Foundation. [Announcement: the issue related the caution of allopurinol in package insert (2009)] [Website in Chinese]. [Internet. Accessed February 17, 2017.] Available from: www.tdrf.org.tw/ch/03_message/mes_02_main.asp?bull_id=3529

16. Fang HY, Hsiao FY, Huang HC, Lin YS, Chen CY, Shieh SH, et al Cost and effectiveness of video-assisted thoracoscopic surgery for clinical stage I non-small cell lung cancer: a population-based analysis. J Thorac Dis 2014;6:1690-6.

17. Mini-Sentinel modular program report: oxicam NSAIDs, modafinil/armodafinil, sulfamothoxazole \& severe cutaneous adverse reaction (SCAR) events. [Internet. Accessed February 17, 2017.] Available from: www.sentinelinitiative.org/sites/default/files/ Drugs/Assessments/Mini-Sentinel_Modular-Program- Report_ MSY2_MP3t4_Oxicam-NSAIDs-Modafinil-Armodafinil-SMZSCAR_1.pdf

18. Kim SC, Newcomb C, Margolis D, Roy J, Hennessy S. Severe cutaneous reactions requiring hospitalization in allopurinol initiators: a population-based cohort study. Arthritis Care Res 2013;65:578-84

19. Cao ZH, Wei ZY, Zhu QY, Zhang JY, Yang L, Qin SY, et al. HLA-B*58:01 allele is associated with augmented risk for both mild and severe cutaneous adverse reactions induced by allopurinol in Han Chinese. Pharmacogenomics 2012;13:1193-201.

20. Dong D, Tan-Koi WC, Teng GG, Finkelstein E, Sung C. Cost-effectiveness analysis of genotyping for HLA-B*5801 and an enhanced safety program in gout patients starting allopurinol in Singapore. Pharmacogenomics 2015;16:1781-93.

21. Saokaew S, Tassaneeyakul W, Maenthaisong R, Chaiyakunapruk N. Cost-effectiveness analysis of HLA-B*5801 testing in preventing allopurinol-induced SJS/TEN in Thai population. PLoS One 2014;9:e94294.

22. Hall G. The clinical application of Bayes' theorem. Lancet 1967;2:555-7.

23. Chohan S. Safety and efficacy of febuxostat treatment in subjects with gout and severe allopurinol adverse reactions. J Rheumatol 2011;38:1957-9.

24. Masbernard A, Giudicelli CP. Ten years' experience with benzbromarone in the management of gout and hyperuricaemia. S Afr Med J 1981;59:701-6.

25. Tsuchiya A, Ikeda S, Ikegami N, Nishimura S, Sakai I, Fukuda T, et al. Estimating an EQ-5D population value set: the case of Japan. Health Econ 2002;11:341-53.

26. Teerawattananon K, Iewsakul S, Yenjitr C, Ausayakhun S, Yenjitr W, Mugford M, et al. Economic evaluation of treatment 
administration strategies of ganciclovir for cytomegalovirus retinitis in HIV/AIDS patients in Thailand: a simulation study.

Pharmacoeconomics 2007;25:413-28.

27. Travers K, Martin A, Khankhel Z, Boye KS, Lee LJ. Burden and management of chronic kidney disease in Japan: systematic review of the literature. Int J Nephrol Renovasc Dis 2013;6:1-13.

28. Becker MA, Schumacher HR, Espinoza LR, Wells AF, MacDonald $\mathrm{P}$, Lloyd E, et al. The urate-lowering efficacy and safety of febuxostat in the treatment of the hyperuricemia of gout: the CONFIRMS trial. Arthritis Res Ther 2010;12:R63.

29. Becker MA, Schumacher HR Jr, Wortmann RL, MacDonald PA, Eustace D, Palo WA, et al. Febuxostat compared with allopurinol in patients with hyperuricemia and gout. N Engl J Med 2005;353:2450-61.

30. Richette P, Doherty M, Pascual E, Barskova V, Becce F, Castañeda-Sanabria J, et al. 2016 updated EULAR evidence-based recommendations for the management of gout. Ann Rheum Dis 2017;76:29-42.

31. Lee MH, Graham GG, Williams KM, Day RO. A benefit-risk assessment of benzbromarone in the treatment of gout. Was its withdrawal from the market in the best interest of patients? Drug Saf 2008;31:643-65.

32. Shu CC, Lee CH, Lee MC, Wang JY, Yu CJ, Lee LN. Hepatotoxicity due to first-line anti-tuberculosis drugs: a five-year experience in a Taiwan medical centre. Int J Tuberc Lung Dis 2013;17:934-9.

33. Kao WY, Su CW, Huang YS, Chou YC, Chen YC, Chung WH, et al. Risk of oral antifungal agent-induced liver injury in Taiwanese. Br J Clin Pharmacol 2014;77:180-9.

34. Hamburger M, Baraf HS, Adamson TC 3rd, Basile J, Bass L, Cole B, et al; European League Against Rheumatism. 2011

Recommendations for the diagnosis and management of gout and hyperuricemia. Postgrad Med 2011;123 Suppl 1:3-36.

35. Jordan KM, Cameron JS, Snaith M, Zhang W, Doherty M, Seckl J, et al; British Society for Rheumatology and British Health Professionals in Rheumatology Standards, Guidelines and Audit Working Group (SGAWG). British Society for Rheumatology and British Health Professionals in Rheumatology guideline for the management of gout. Rheumatology 2007;46:1372-4.

36. Frequency of adverse drug reactions. In: Guidelines for preparing core clinical safety information on drugs. Report of CIOMS Working Group III. Geneva: World Health Organization; 1995.

37. National Health Insurance Administration, Ministry of Health and Welfare. Reimbursed drug price. [Internet. Access February 10,
2017.] Available from: www.nhi.gov.tw/Query/query 1.aspx? menu=20\&menu_id=831\&WD_ID=831

38. Yang CY, Chen CH, Deng ST, Huang CS, Lin YJ, Chen YJ, et al. Allopurinol use and risk of fatal hypersensitivity reactions: a nationwide population-based study in Taiwan. JAMA Intern Med 2015;175:1550-7.

39. Economic Forecast Section, Department of Statistics, Directorate General of Budget, Accounting \& Statistics (DGBAS). Taiwan's real gross domestic product (GDP) per capita. [Internet. Accessed February 10, 2017.] Available from: www.dgbas.gov.tw/point.asp?index $=1$

40. Li L, Yang C, Zhao Y, Zeng X, Liu F, Fu P. Is hyperuricemia an independent risk factor for new-onset chronic kidney disease?: A systematic review and meta-analysis based on observational cohort studies. BMC Nephrol 2014;15:122.

41. Paisansinsup T, Breitenstein MK, Schousboe JT. Association between adverse reactions to allopurinol and exposures to high maintenance doses: implications for management of patients using allopurinol. J Clin Rheumatol 2013;19:180-6.

42. Vázquez-Mellado J, Morales EM, Pacheco-Tena C, Burgos-Vargas R. Relation between adverse events associated with allopurinol and renal function in patients with gout. Ann Rheum Dis 2001;60:981-3.

43. Zhong H, Zhou Z, Wang H, Niu J, Chen W, Song Z, et al. Prevalence of cutaneous adverse drug reactions in Southwest China: an 11-year retrospective survey on in-patients of a dermatology ward. Dermatitis 2012;23:81-5.

44. Otto MA. Febuxostat tied to hypersensitivity reactions: reports include two cases of Stevens-Johnson syndrome. Family Practice News 2010 June 15. [Internet. Accessed February 17, 2017.] Available from: www.mdedge.com/familypracticenews/article/31315 /rheumatology/febuxostat-tied-hypersensitivity-reactions-reports

45. Abeles AM. Febuxostat hypersensitivity. J Rheumatol 2012;39:659.

46. Chang YS, Huang FC, Tseng SH, Hsu CK, Ho CL, Sheu HM. Erythema multiforme, Stevens-Johnson syndrome, and toxic epidermal necrolysis: acute ocular manifestations, causes, and management. Cornea 2007;26:123-9.

47. Saeed H, Mantagos IS, Chodosh J. Complications of Stevens-Johnson syndrome beyond the eye and skin. Burns 2016;42:20-7.

48. Park DJ, Kang JH, Lee JW, Lee KE, Wen L, Kim TJ, et al. Cost-effectiveness analysis of HLA-B5801 genotyping in the treatment of gout patients with chronic renal insufficiency in Korea. Arthritis Care Res 2015;67:280-7. 